\title{
A method for uncertainty and sensitivity analysis in fuel performance codes
}

\author{
Dantas A.C., Silva, A. T. \\ Instituto de Pesquisas Energéticas e Nucleares, 05508-000, São Paulo, São Paulo, Brasil \\ anadantas.ac@gmail.com \\ teixeira@ipen.br
}

\begin{abstract}
The present study proposes a method for the execution of uncertainty and sensitivity analysis on TRANSURANUS code, adapted for the use of stainless steel AISI-348 as the cladding material for a PWR reactor fuel rod, thus allowing to determine which input data are more relevant to the TRANSURANUS models, as well as a confidence interval for the results. The analysis was made through Monte Carlo sampling, where input values related to the geometry and composition of the fuel rod were taken from a normal distribution truncated around fabrication tolerance values. The generated samples were used as TRANSURANUS input data, and after numerous executions of the code, the results pertaining to the fuel center line temperature, fuel rod inner pressure and cladding strains were used to obtain a confidence interval and to make a variance-based sensitivity analysis, showing that the models used in TRANSURANUS are additive in nature, and input interactions are not relevant to the code.
\end{abstract}

Keywords: fuel performance code, sensitivity analysis, uncertainty analysis, confidence intervals. 


\section{INTRODUCTION}

The licensing process of nuclear reactors is subject to the presenting of calculations and experimental data showing that reactor operation under normal conditions occurs within safety bounds. Calculations such as these can only be performed numerically using fuel performance codes.

When designing a simulation to be run in such codes, a virtual experiment can be conservative, choosing the "worst case" of each variable involved, or realistic, choosing design values for variables and most probable values for each parameter. For many years, conservative analysis was preferred, but the perfecting of fuel performance codes has made predictions of realistic models more and more reliable, opening a possibility for new and daring reactor designs.

Of course, despite the increasing reliability of fuel performance codes, calculations remain subject to uncertainties: unknown values of code parameters, parameters subject to natural variation, uncertainties pertaining to numerical solutions, to the fabrication of fuel rod components and to the model itself. Therefore, in order to make a realistic analysis of a nuclear reactor it is necessary to establish a confidence interval for the results that takes into account the uncertainties present in the model [1].

Another useful tool for model analysis is sensitivity analysis, which attributes a relative importance to each of the uncertain model inputs. The present work describes a method for uncertainty analysis in fuel performance codes, as well as sensitivity analysis by variance decomposition [2]. Such analysis will be applied to the fabrication parameters of a stainless steel clad fuel rod from a hypothetical pressurized water reactor (PWR). At the conclusion of this study, it is expected that a confidence interval for outputs related to safety criteria - fuel center line temperature, fuel rod inner pressure and cladding creep strain - will be obtained, as well as an attribution of importance to each of the input factors.

\section{MATERIALS AND METHODS}

The fuel performance code chosen for this study was TRANSURANUS [3], a code developed by the Institute of Transuranium Elements in Europe, focused on the thermal and mechanical analysis of fuel rods in nuclear reactors. Following the Fukushima nuclear accident, a search began for improved, 
accident resistant cladding materials [4]. The version of the TRANSURANUS code chosen for this analysis was the version developed by Giovedi et al (2019) which includes a library of thermal and mechanical properties of stainless steel AISI-348, chosen as the cladding material of the hypothetical fuel rod due to it's structural advantages, such a greater resistance to corrosion when compared to other stainless steel claddings [5,6].

In order to perform a global uncertainty and sensitivity analysis, input values considered uncertain are chosen from a random distribution that represents the behavior of said variable, TRASURANUS is run with sampled values and relevant output is saved. This process is repeated for $\mathrm{N}$ values of each input parameter. In order to do that, TRANSURANUS is coupled with GNU Octave, a high level language focused on numerical solutions. GNU Octave scripts are used to generate $\mathrm{N}$ samples of each input parameter according to chosen distribution, automatize the generation of new TRANSURANUS input files, run TRANSURANUS and extract and save data from TRASURANUS output files, which is then analyzed $[7,8,9]$.

\section{Sensitivity analysis}

The method chosen for realization of sensitivity analysis was variance decomposition. By this method, a sensitivity index $S_{i}$ could be defined as:

$$
S_{i}=\frac{V\left[E\left(Y / X_{i}\right)\right]}{V(Y)}
$$

Where $\mathrm{E}\left(\mathrm{Y} \mid \mathrm{X}_{\mathrm{i}}\right)$ is the expectation value of the result vector $\mathrm{Y}$ when the variable $\mathrm{X}_{\mathrm{i}}$ is kept fixed. This should be calculated for each of the $\mathrm{N}$ values $\mathrm{X}_{\mathrm{i}}$ can take. Considering a total of $\mathrm{k}$ uncertain inputs, the calculation of sensitivity indices using Equation (1) would require $\mathrm{N}^{\mathrm{k}}$ operations, which can be extremely computationally costly. Thus, the method developed by Saltelli et al (2008) [2] is chosen. By this method, the number of operations can be reduced to $\mathrm{N}(\mathrm{k}+2)$, a much more manageable quantity.

In this method, two groups of samples, $\mathrm{A}$ and $\mathrm{B}$, are generated in the form of matrices of $\mathrm{N}$ lines and $\mathrm{k}$ columns corresponding to the uncertain inputs. The model is applied to A and B, producing the result vectors $\mathrm{y}_{\mathrm{A}}$ and $\mathrm{y}_{\mathrm{B}}$. Then matrices $\mathrm{A}$ and $\mathrm{B}$ are recombined into a matrix $\mathrm{C}_{\mathrm{i}}$ where the $\mathrm{i}$-th column 
of $B$ is substituted by the $\mathrm{i}$-th column of $\mathrm{A}$. The model is applied to $\mathrm{C}_{\mathrm{i}}$, generating a $\mathrm{y}_{\mathrm{Ci}}$ result vector. In possession of these quantities, sensitivity indices $S_{i}$ are given by:

$$
S_{i}=\frac{y_{A} \cdot y_{C_{i}}-f_{0}^{2}}{y_{A} \cdot y_{A}-f_{0}^{2}}=\frac{(1 / N) \sum_{j=1}^{N} \quad y_{A}^{(j)} y_{C_{i}}^{(j)}-f_{0}^{2}}{(1 / N) \sum_{\mathrm{j}=1}^{N}\left(y_{a}^{(j)}\right)^{2}-f_{0}^{2}}
$$

where

$$
f_{0}^{2}=\left(\frac{1}{N} \sum_{\mathrm{j}=1}^{N} \quad y_{A}^{(j)}\right)^{2}
$$

To account for input interactions, another quantity of interest in the index of total effects, which quantifies the variance caused by the variation of every variable except for $\mathrm{X}_{\mathrm{i}}$, defined as:

$$
S_{T_{i}}=1-\frac{V\left[E\left(Y / X_{(i)}\right)\right]}{V(Y)}
$$

and calculated by the Saltelli method as:

$$
S_{T_{i}}=1-\frac{y_{B} \cdot y_{C_{i}}-f_{0}^{2}}{y_{A} \cdot y_{A}-f_{0}^{2}}=1-\frac{(1 / N) \sum_{\mathrm{j}=1}^{N} \quad y_{B}^{(j)} y_{C_{i}}^{(j)}-f_{0}^{2}}{(1 / N) \sum_{\mathrm{j}=1}^{N}\left(y_{a}^{(j)}\right)^{2}-f_{0}^{2}}
$$

When input interactions are relevant to the model, $\mathrm{S}_{\mathrm{Ti}}>\mathrm{S}_{\mathrm{i}}$, the difference accounting for higher order effects. In additive models, this should not be the case, with $\mathrm{S}_{\mathrm{Ti}}=\mathrm{S}_{\mathrm{i}}$ and the sum of first order sensitivity indices equals to 1 .

By definition, the indices given by Equations (1) and (3) are positive. Since Equations (2) and (4) operate based on random sampling, their results may be negative (though when $\mathrm{N}$ is sufficiently large these values should be equal to zero when rounded). In order to minimize statistical error, multiple simulations were run, and their results averaged. That also allowed for the calculation of standard error pertaining to each index, making it possible to determine which indices were statistically equal to zero. In this work, 10 simulations with $\mathrm{N}=2000$ were run. 
As additional evidence pertaining to the additivity of the model and relative importance of input parameters, Spearman's rank-order correlations between results yA and each column of inputs were calculated. Spearman correlation is equal to the Pearson correlation, given by Equation (5), but using the ranks of data (highest value, $2^{\text {nd }}$ highest, and so forth) rather than their absolute values.

$$
\rho=\frac{\operatorname{cov}(x, y)}{\sigma_{x} \sigma_{y}}
$$

Where $\mathrm{x}$ and $\mathrm{y}$ are sets of data (or sets of ranks of data), cov is the covariance between data sets, and $\sigma$ is the standard deviation of the considered variables [10].

Studying the correlation between ranks of data rather than data itself makes Spearman correlation indexes useful for assessment of monotonic relationships, whether linear or not. An example of usage of Spearman rank-order correlations in sensitivity analysis can be found in reference [9].

\section{Uncertainty analysis}

As mentioned in the above section, ten simulations with 2000 samples each were run. Taking the $\mathrm{y}_{\mathrm{A}}$ results from each simulation, a total of 20,000 points of data are obtained, which are used to generate histograms of the distribution of each of the three outputs considered. Qualitative analysis of the histograms allows to verify if the general behavior matches that of a normal distribution, and a gaussian is fitted to the histogram data points to provide additional confirmation. The mean and standard deviation obtained from the histogram are compared to the mean and standard deviation calculated straight from the data.

From this mean and standard deviation it is possible to calculate the confidence intervals of each of the considered output parameters. According to Wilk's formula, to guarantee that a quantity $\alpha$ of the samples are within a $\beta$ confidence interval, a minimum of $n$ samples are necessary, where:

$$
1-\alpha^{n}-n(1-\alpha) \alpha^{n-1} \geqslant \beta
$$

For $\alpha=0.95$ ( $95 \%$ of the samples) and $\beta=0.95$ (95\% confidence interval), at least 93 samples are required. As the number of samples available greatly surpasses that, the calculated interval has at least $95 \%$ confidence. 


\section{Input values and reactor power cycles}

Thirteen input parameters were considered subject to fabrication uncertainties. Twelve of them represent variables of the TRANSURANUS code, their names and physical meaning described in Table 1 . The thirteenth variable described in the table refers to a ratio to be multiplied by a vector of code variables.

Table 1: Names of TRANSURANUS input parameters subject to variation.

\begin{tabular}{|c|c|}
\hline Variable name & Description \\
\hline RAH & Outer radius of the cladding [mm] \\
\hline pi0ein & Filling gas pressure $[\mathrm{MPa}]$ \\
\hline enriU235 & Initial concentration ("enrichment") of U-235 (/) \\
\hline ozum0 & Average ratio between oxide and metal atoms (/) \\
\hline RAB & Outer radius of the fuel [mm] \\
\hline prodis & Fraction of dish volume to pellet volume (/) \\
\hline korngr & Fabrication grain size diameter in the fuel $[\mathrm{mm}]$ \\
\hline por000 & Average fabrication porosity of the fuel (/) \\
\hline denpor & Minimum porosity at the end of densification. \\
\hline RIH & Inner radius of the cladding [mm] \\
\hline uplvg & Lower plenum volume $\left[\mathrm{mm}^{3}\right]$ \\
\hline openpor & Open porosity (/) \\
\hline hhrate & Ratio to be multiplied by the active length of the fuel. \\
\hline
\end{tabular}

As these input parameters refer to fabrication parameters, normal distribution was considered as an adequate representation. A maximum and minimum values were also defined, at which the distribution was truncated, representing the fact that fabricated pieces that do not fit design criteria are rejected and not used in the reactor. The nominal values, standard errors and maximum and minimum values were defined based on those of commercial PWR reactors, and are shown in Table 2. 
Table 2: Values of TRANSURANUS input variables.

\begin{tabular}{ccccc}
\hline Variable name & Mean & Standard deviation & Minimum & Maximum \\
\hline RAH & 4.900 & 0.025 & 4.875 & 4.925 \\
pi0ein & 2.76 & $-0.35 /+0$ & 2.41 & 2.76 \\
enriU235 & 0.0430 & 0.0004 & 0.0426 & 0.0434 \\
ozum0 & 2.00 & 0.02 & 2.00 & 2.02 \\
RAB & 4.245 & 0.005 & 4.240 & 4.250 \\
prodis & 0.018 & 0.002 & 0.010 & 0.030 \\
korngr & 0.0061 & 0.0080 & 0.0040 & 0.0250 \\
por000 & 0.060 & 0.009 & 0.051 & 0.069 \\
denpor & 0.0381 & 0.0080 & 0.0270 & 0.0550 \\
RIH & 4.30 & 0.02 & 4.28 & 4.32 \\
uplvg & 210.10 & 68.99 & 73.05 & 349.02 \\
openpor & 0.04 & 0.04 & 0 & 0.04 \\
hhrate & 1.00 & 0.01 & 1.00 & 1.01 \\
\hline
\end{tabular}

As for the boundary conditions of the simulation, the hypothetical reactor was subject to a power cycle where, during the beginning of life of the fuel, the linear rod power was abruptly raised from $30 \%$ to $100 \%$, being kept in full power for ten days at a time, and descending abruptly back to the $30 \%$ mark. This cycle, shown in Figure 1, is repeated five times, after which operation is kept at a $30 \%$ level until 26,400 hours (approximately 3 years) of operation are reached. 


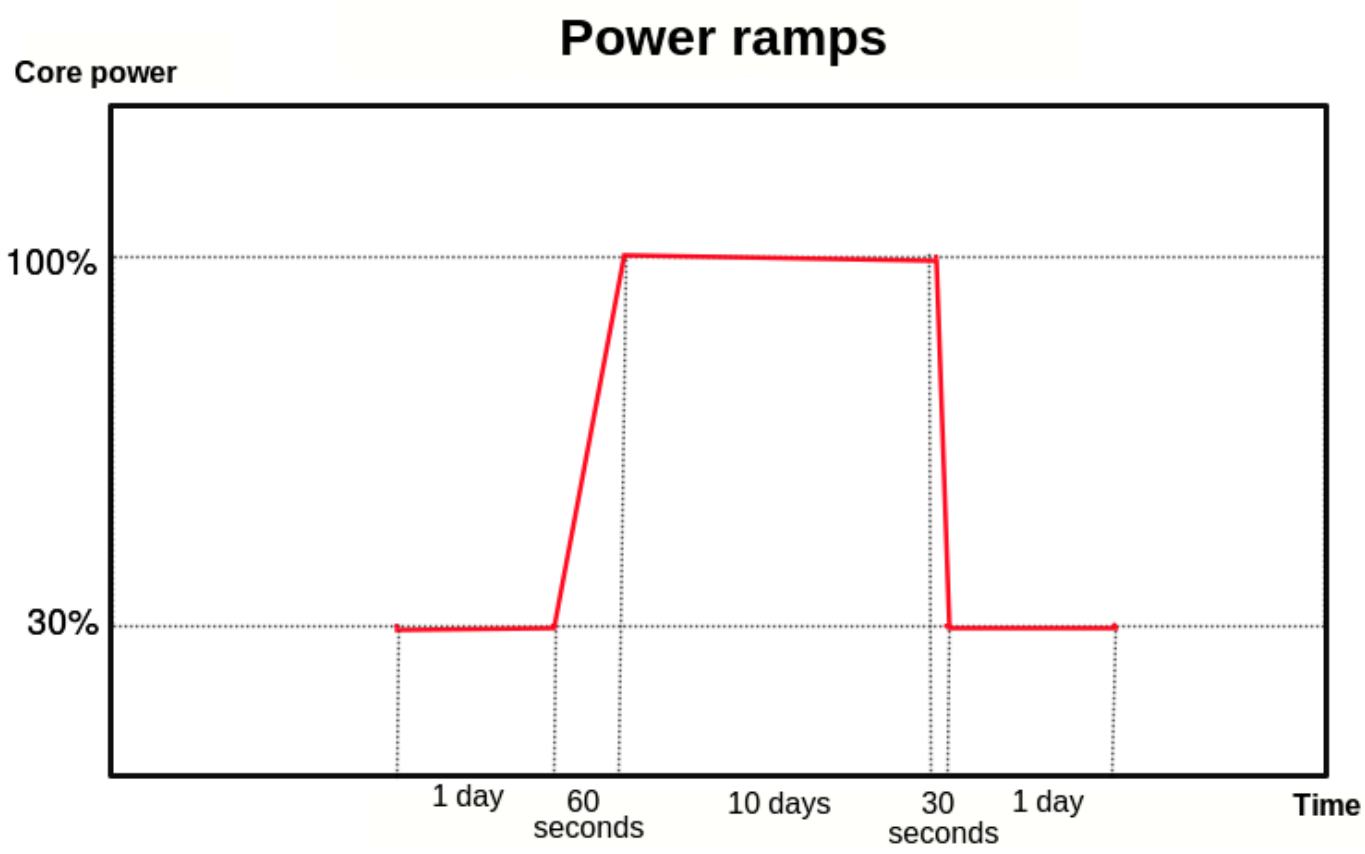

Figure 1: Operation power cycles.

\section{RESULTS AND DISCUSSION}

At the end of the ten runs of simulations, ten tables of Spearman ranking correlations were generated, one for each A sample group. Only two of such will be shown here, in Tables 3 and 4, in order to exemplify the qualitative likeness of the obtained results. The highest correlation indices in absolute values are shown in bold italics to facilitate their location in both tables. 
Table 3: Spearman ranking correlations obtained from simulation 1

\begin{tabular}{cccc}
\hline & \multicolumn{2}{c}{ Spearman ranking correlations } \\
\cline { 2 - 4 } Uncertain Inputs & $\begin{array}{c}\text { Fuel centerline } \\
\text { temperature }\end{array}$ & Fuel rod pressure & Cladding creep strain \\
\hline RAH & 0.0323 & 0.0243 & $\mathbf{- 0 . 5 4 5 8}$ \\
pi0ein & -0.0133 & $\mathbf{0 . 9 5 8 7}$ & $\mathbf{- 0 . 5 1 2 0}$ \\
enriU235 & -0.0005 & -0.0074 & 0.0065 \\
ozum0 & 0.0135 & 0.0140 & -0.0124 \\
RAB & $\mathbf{- 0 . 1 9 6 7}$ & -0.0572 & 0.0212 \\
prodis & 0.0101 & $\mathbf{0 . 1 3 5 5}$ & -0.0418 \\
korngr & -0.0009 & 0.0031 & 0.0027 \\
por000 & $\mathbf{0 . 5 2 5 1}$ & -0.0036 & $\mathbf{0 . 3 2 1 4}$ \\
denpor & $\mathbf{- 0 . 4 4 0 6}$ & 0.0419 & $\mathbf{- 0 . 4 5 3 8}$ \\
RIH & $\mathbf{0 . 6 5 0 9}$ & $\mathbf{0 . 2 4 6 8}$ & $\mathbf{0 . 3 0 0 9}$ \\
uplvg & -0.0416 & 0.0104 & -0.0476 \\
openpor & 0.0006 & 0.0209 & -0.0161 \\
hhrate & 0.0053 & -0.0340 & 0.0361 \\
\hline Sum of $\rho^{2}$ & 0.9354 & 1.0059 & 0.9661 \\
\hline
\end{tabular}


Table 4: Spearman ranking correlations obtained from simulation 6

Spearman ranking correlations

\begin{tabular}{cccc}
\cline { 2 - 4 } Uncertain Inputs & $\begin{array}{c}\text { Fuel centerline } \\
\text { temperature }\end{array}$ & Fuel rod pressure & Cladding creep strain \\
\hline RAH & 0.0093 & 0.0090 & $\mathbf{- 0 . 5 4 0 7}$ \\
pi0ein & -0.0011 & $\mathbf{0 . 9 5 8 1}$ & $\mathbf{- 0 . 4 9 2 6}$ \\
enriU235 & 0.0222 & 0.0129 & 0.0260 \\
ozum0 & 0.0252 & 0.0105 & 0.0112 \\
RAB & $\mathbf{- 0 . 1 5 6 8}$ & -0.0590 & 0.0332 \\
prodis & 0.0162 & $\mathbf{0 . 1 4 6 4}$ & -0.0614 \\
korngr & -0.0260 & 0.0040 & 0.0106 \\
por000 & $\mathbf{0 . 5 5 0 0}$ & -0.0446 & $\mathbf{0 . 3 5 0 5}$ \\
denpor & $\mathbf{- 0 . 4 6 1 1}$ & -0.0141 & $\mathbf{- 0 . 4 4 4 2}$ \\
RIH & $\mathbf{0 . 6 6 2 4}$ & $\mathbf{0 . 2 5 2 2}$ & $\mathbf{0 . 3 3 6 3}$ \\
uplvg & 0.0132 & -0.0280 & 0.0150 \\
openpor & 0.0316 & 0.0069 & 0.0327 \\
hhrate & 0.0118 & -0.0302 & 0.0386 \\
\hline Sum of $\rho^{2}$ & 0.9819 & 1.0109 & 0.9769 \\
\hline & & & \\
\hline
\end{tabular}

The input factors considered relevant agree throughout the ten simulations: outer radius of the fuel and inner radius of the cladding (which together define the gap between fuel and cladding) as well as fabrication porosity and porosity at the end of densification are the most important factors for fuel center line temperature; filling gas pressure, ratio between dish and pellet volume and inner radius of the cladding are relevant to pressure of the fuel rod; and cladding creep strain is mostly affected by cladding outer and inner radius (cladding thickness), filling gas pressure, fabrication porosity, porosity at the end of densification and fuel pellet radius.

For correlation indices too close to zero (inferior to 0.05), signs vary between positive and negative, accounting for the statistical nature of the analysis. The sums of rho ${ }^{2}$ throughout the simulations show that TRANSURANUS model for the chosen outputs are strongly additive, though 
variance as to how close they are to the unity makes it so that they can't be declared - by this method alone - perfectly additive.

The sensitivity indices calculated by Equation (2) do not agree so well among themselves. Tables 5 and 6 were chosen from the ten sets of calculated indices to exemplify their discrepancies. Because of these discrepancies, no values were highlighted as most important.

Table 5: First order sensitivity indices from simulation 1

Sensitivity indices

\begin{tabular}{cccc}
\cline { 2 - 3 } Uncertain Inputs & $\begin{array}{c}\text { Fuel centerline } \\
\text { temperature }\end{array}$ & Fuel rod pressure & Cladding creep strain \\
\hline RAH & -0.0619 & 0.0490 & 0.3438 \\
pi0ein & 0.0082 & 0.9122 & 0.2197 \\
enriU235 & -0.1514 & 0.0575 & 0.0433 \\
ozum0 & -0.1579 & 0.0437 & 0.0433 \\
RAB & -0.0889 & 0.0406 & 0.0399 \\
prodis & -0.1075 & 0.0688 & 0.0396 \\
korngr & -0.1141 & 0.0487 & 0.0433 \\
por000 & 0.2593 & 0.0803 & 0.1424 \\
denpor & 0.2798 & 0.0615 & 0.2280 \\
RIH & 0.2804 & 0.0942 & 0.1725 \\
uplvg & -0.0112 & 0.0614 & 0.0369 \\
openpor & -0.1461 & 0.0431 & 0.0433 \\
hhrate & -0.0580 & 0.0537 & 0.0431 \\
\hline Sum of $\mathrm{S}_{\mathrm{i}}$ & -0.0693 & 1.6147 & 1.4391 \\
\hline
\end{tabular}


Table 6: First order sensitivity indices from simulation 6 sensitivity indices

\begin{tabular}{cccc}
\hline & \multicolumn{3}{c}{ sensitivity indices } \\
\cline { 2 - 4 } Uncertain Inputs & $\begin{array}{r}\text { Fuel centerline } \\
\text { temperature }\end{array}$ & Fuel rod pressure & Cladding creep strain \\
\hline RAH & 0.0391 & 0.0096 & 0.3717 \\
pi0ein & 0.1518 & 0.8762 & 0.3525 \\
enriU235 & 0.1175 & 0.0043 & 0.0567 \\
ozum0 & 0.1638 & 0.0036 & 0.0566 \\
RAB & 0.0108 & 0.0105 & 0.0521 \\
prodis & -0.0725 & 0.0066 & 0.0549 \\
korngr & 0.0266 & 0.0051 & 0.0567 \\
por000 & 0.3664 & 0.0009 & 0.1624 \\
denpor & 0.3220 & 0.0305 & 0.2540 \\
RIH & 0.6679 & 0.0816 & 0.1291 \\
uplvg & 0.0089 & 0.0011 & 0.0528 \\
openpor & 0.1806 & 0.0043 & 0.0566 \\
hhrate & 0.0182 & 0.0070 & 0.0563 \\
\hline Sum of $\mathrm{S}_{\mathrm{i}}$ & 2.0011 & 1.0413 & 1.7124 \\
\hline
\end{tabular}

The first noteworthy feature of Tables 5 and 6 is the presence of negative numbers with high absolute value. As those indices are obtained by statistical methods, fluctuations around zero are to be expected, but Table 5 shows multiple values inferior than -0.10 , greatly compromising the premise that the sum of indices $S_{i}$ in additive models should be equal to 1. The comparison between the first three lines of the temperature column in both tables shows that simulations cannot agree about which indices are nearly zero and which ones are positive or negative. For the indices considered relevant by the correlations, agreement was better, though not perfect, demonstrating the need of averaging the results of simulations as discussed in the previous section.

Table 7 shows the averaged vales of first order sensitivity indices, with standard deviation obtained from the variance of the ten results. These standard deviations were used to define which values are statistically different from zero, such values are presented in bold italics. 
Table 7: Averaged first order sensitivity indices

\section{sensitivity indices}

\begin{tabular}{cccc}
\cline { 2 - 4 } Uncertain Inputs & $\begin{array}{c}\text { Fuel centerline } \\
\text { temperature }\end{array}$ & Fuel rod pressure & Cladding creep strain \\
\hline RAH & $0.0512 \pm 0.1188$ & $0.0038 \pm 0.0294$ & $\mathbf{0 . 3 0 3 6} \pm \mathbf{0 . 0 5 3 3}$ \\
pi0ein & $0.0590 \pm 0.1168$ & $\mathbf{0 . 9 1 6 7} \pm \mathbf{0 . 0 2 1 3}$ & $\mathbf{0 . 2 5 3 8} \pm \mathbf{0 . 0 5 5 8}$ \\
enriU235 & $-0.0040 \pm 0.1009$ & $0.0025 \pm 0.0309$ & $0.0151 \pm 0.0562$ \\
ozum0 & $0.0096 \pm 0.0966$ & $-0.0005 \pm 0.0248$ & $0.0151 \pm 0.0562$ \\
RAB & $0.0306 \pm 0.0718$ & $0.0095 \pm 0.0284$ & $0.0134 \pm 0.0565$ \\
prodis & $0.0070 \pm 0.1181$ & $0.0146 \pm 0.0307$ & $0.0175 \pm 0.0577$ \\
korngr & $0.0020 \pm 0.0805$ & $0.0020 \pm 0.0270$ & $0.0151 \pm 0.0562$ \\
por000 & $\mathbf{0 . 2 7 0 7} \pm \mathbf{0 . 1 2 8 3}$ & $-0.0033 \pm 0.0420$ & $\mathbf{0 . 1 3 6 5} \pm \mathbf{0 . 0 4 6 9}$ \\
denpor & $\mathbf{0 . 2 4 0 1} \pm \mathbf{0 . 0 6 8 0}$ & $-0.0052 \pm 0.0336$ & $\mathbf{0 . 2 1 5 2} \pm \mathbf{0 . 0 4 1 7}$ \\
RIH & $\mathbf{0 . 4 7 7 2} \pm \mathbf{0 . 1 2 9 4}$ & $\mathbf{0 . 0 6 2 7} \pm \mathbf{0 . 0 2 6 3}$ & $\mathbf{0 . 1 3 4 9} \pm \mathbf{0 . 0 6 1 2}$ \\
uplvg & $0.0200 \pm 0.0842$ & $0.0027 \pm 0.0298$ & $0.0152 \pm 0.0540$ \\
openpor & $0.0129 \pm 0.0982$ & $-0.0003 \pm 0.0251$ & $0.0151 \pm 0.0562$ \\
hhrate & $0.0174 \pm 0.0504$ & $0.0008 \pm 0.0270$ & $0.0150 \pm 0.0562$ \\
\hline Sum of $\mathrm{S}_{\mathrm{i}}$ & $1.1936 \pm 0.3605$ & $1.0061 \pm 0.1058$ & $1.1657 \pm 0.1971$ \\
\hline & & & \\
\hline
\end{tabular}

Table 7 is qualitatively closer to the results of correlation indices shown in Tables 3 and 4, though the importance of RAB and prodis was removed. The sum of sensitivity indices now is close to the unity, though a large standard variation makes it hard to declare the model as $100 \%$ additive. Finally, Table 8 shows the averaged total effects indices, which, when compared to the first order indices allow the detection of second order effects. The indices statistically different from zero are shown in bold italics. 
Table 8: Averaged total effects indices

\section{sensitivity indices}

\begin{tabular}{cccc}
\cline { 2 - 4 } Uncertain Inputs & $\begin{array}{c}\text { Fuel centerline } \\
\text { temperature }\end{array}$ & Fuel rod pressure & Cladding creep strain \\
\hline RAH & $-0.0715 \pm 0.1977$ & $-0.0016 \pm 0.0602$ & $\mathbf{0 . 2 8 3 2} \pm \mathbf{0 . 1 1 5 6}$ \\
pi0ein & $-0.0781 \pm 0.2058$ & $\mathbf{0 . 9 1 7 0} \pm \mathbf{0 . 0 3 6 7}$ & $\mathbf{0 . 2 4 0 8} \pm \mathbf{0 . 1 0 8 9}$ \\
enriU235 & $-0.0163 \pm 0.1977$ & $-0.0002 \pm 0.0599$ & $-0.0187 \pm 0.1225$ \\
ozum0 & $-0.0411 \pm 0.1973$ & $0.0026 \pm 0.0533$ & $-0.0187 \pm 0.1225$ \\
RAB & $0.0187 \pm 0.1617$ & $-0.0007 \pm 0.0643$ & $-0.0144 \pm 0.1223$ \\
prodis & $-0.0268 \pm 0.2003$ & $0.0243 \pm 0.0597$ & $-0.0099 \pm 0.1234$ \\
korngr & $-0.0209 \pm 0.1787$ & $0.0000 \pm 0.0555$ & $-0.0187 \pm 0.1225$ \\
por000 & $\mathbf{0 . 3 2 8 8} \pm \mathbf{0 . 2 0 5 2}$ & $0.0062 \pm 0.0618$ & $0.0854 \pm 0.1072$ \\
denpor & $\mathbf{0 . 1 6 5 3} \pm \mathbf{0 . 1 4 3 8}$ & $0.0095 \pm 0.0511$ & $\mathbf{0 . 2 0 1 6} \pm \mathbf{0 . 1 1 2 5}$ \\
RIH & $\mathbf{0 . 4 0 3 2} \pm \mathbf{0 . 2 0 1 7}$ & $\mathbf{0 . 0 5 9 3} \pm \mathbf{0 . 0 5 6 5}$ & $\mathbf{0 . 1 0 0 3} \pm \mathbf{0 . 1 1 1 8}$ \\
uplvg & $-0.0406 \pm 0.1751$ & $-0.0002 \pm 0.0576$ & $-0.0183 \pm 0.1205$ \\
openpor & $-0.0448 \pm 0.1968$ & $0.0023 \pm 0.0541$ & $-0.0187 \pm 0.1225$ \\
hhrate & $-0.0374 \pm 0.1395$ & $0.0014 \pm 0.0553$ & $-0.0185 \pm 0.1224$ \\
\hline & & &
\end{tabular}

Careful comparison between Tables 7 and 8 reveals that some total effects indices are inferior to their respective first order sensitivity indices. In theory this should not occur, but it can also be credited to statistical fluctuations. In any case, comparison between indices does not allow to identify second order effects.

As for the uncertainty analysis, Table 9 shows the obtained mean, average and confidence intervals for the considered outputs, while Figures 2, 3 and 4 show the Gaussian curves fitted to their respective histograms. 
Table 9: Results of uncertainty analysis

\begin{tabular}{ccc}
\hline Output & $\begin{array}{c}\text { Mean and standard } \\
\text { deviation }\end{array}$ & Confidence interval \\
\hline Fuel center line temperature $\left({ }^{\circ} \mathrm{C}\right)$ & $1296.1 \pm 56.6$ & {$[1185.0,1407.1]$} \\
Fuel rod pressure $(\mathrm{MPa})$ & $5.16 \pm 0.20$ & {$[4.77,5.56]$} \\
Cladding creep strain $(\%)$ & $0.0137 \pm 0.0005$ & {$[0.0128,0,0147]$} \\
\hline
\end{tabular}

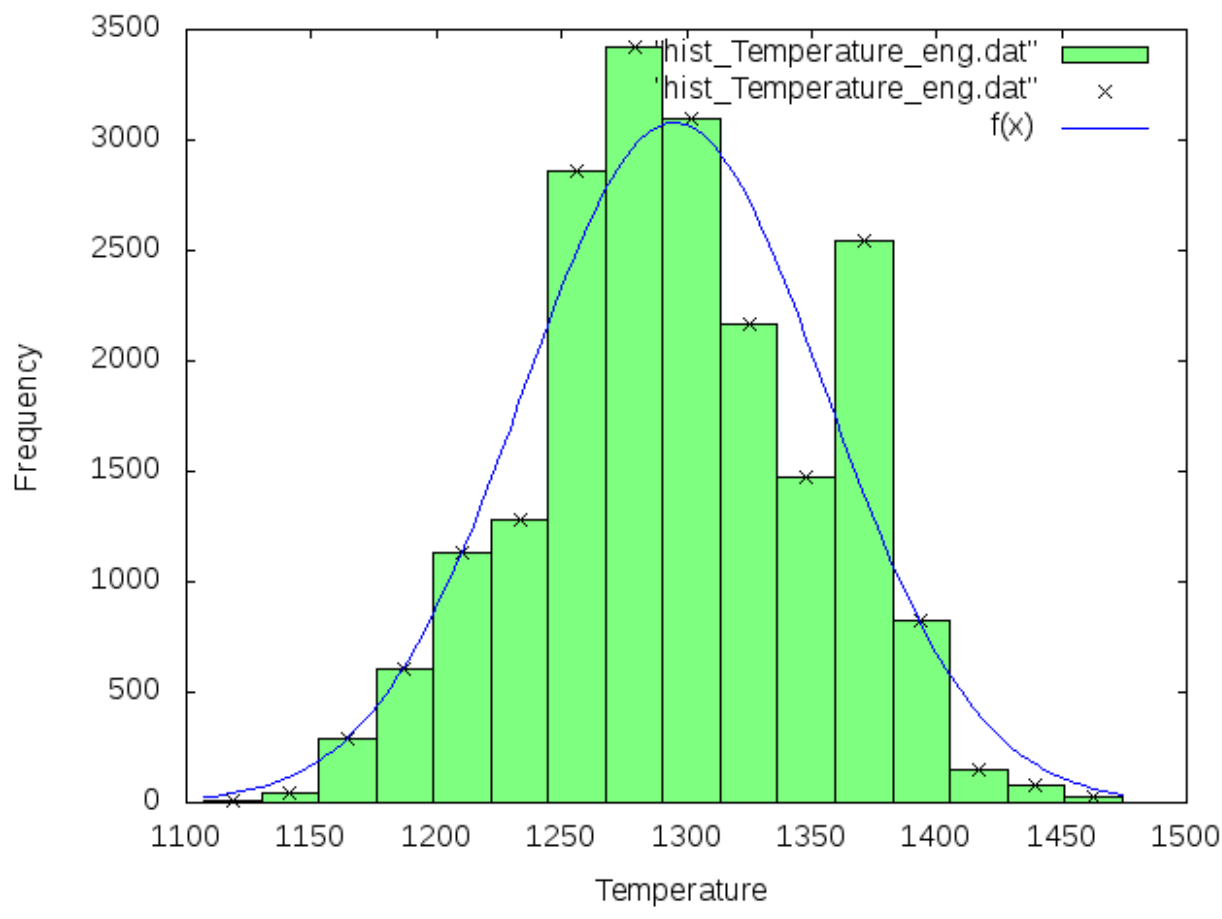

Figure 2: Distribution of fuel center line temperature results $\left({ }^{\circ} \mathrm{C}\right)$. 


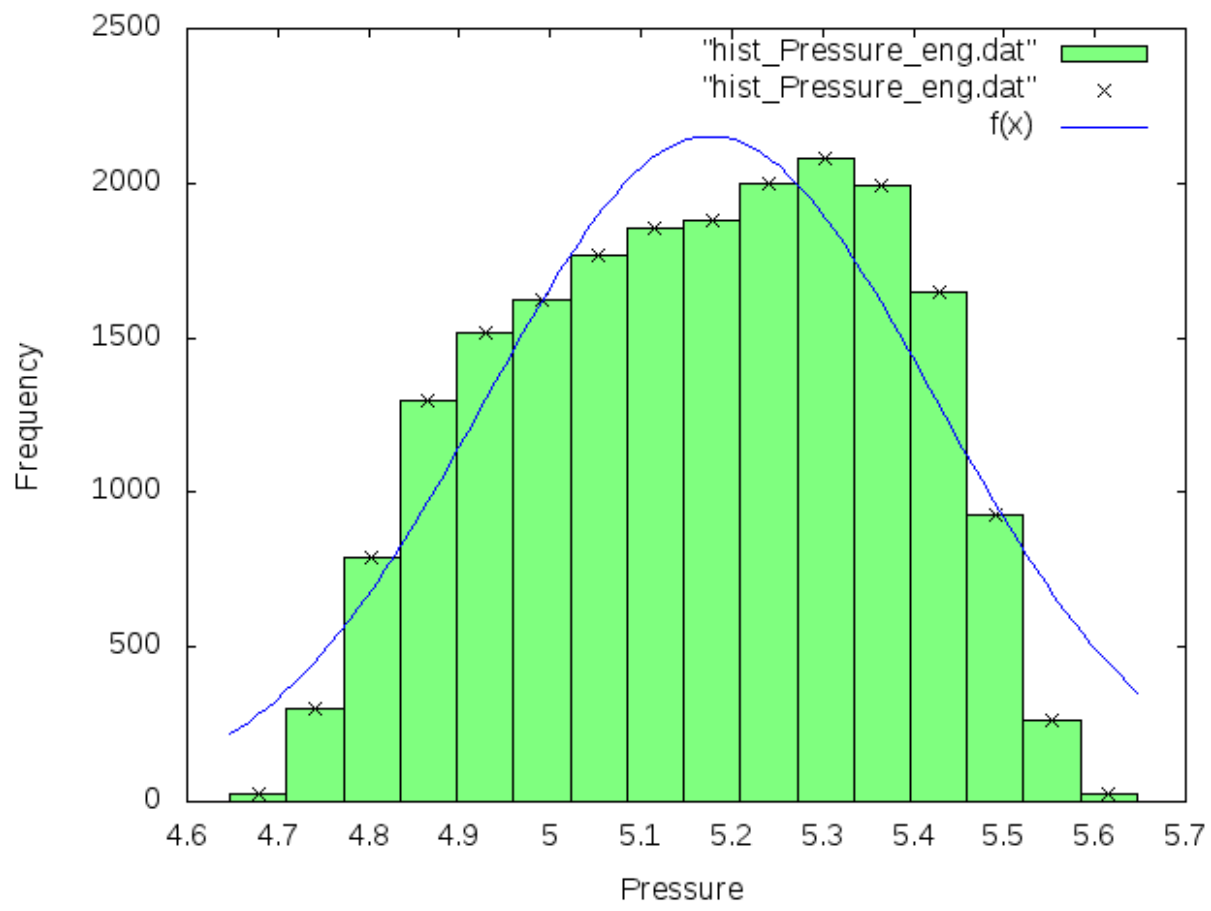

Figure 3: Distribution of fuel rod inner pressure results (MPa).

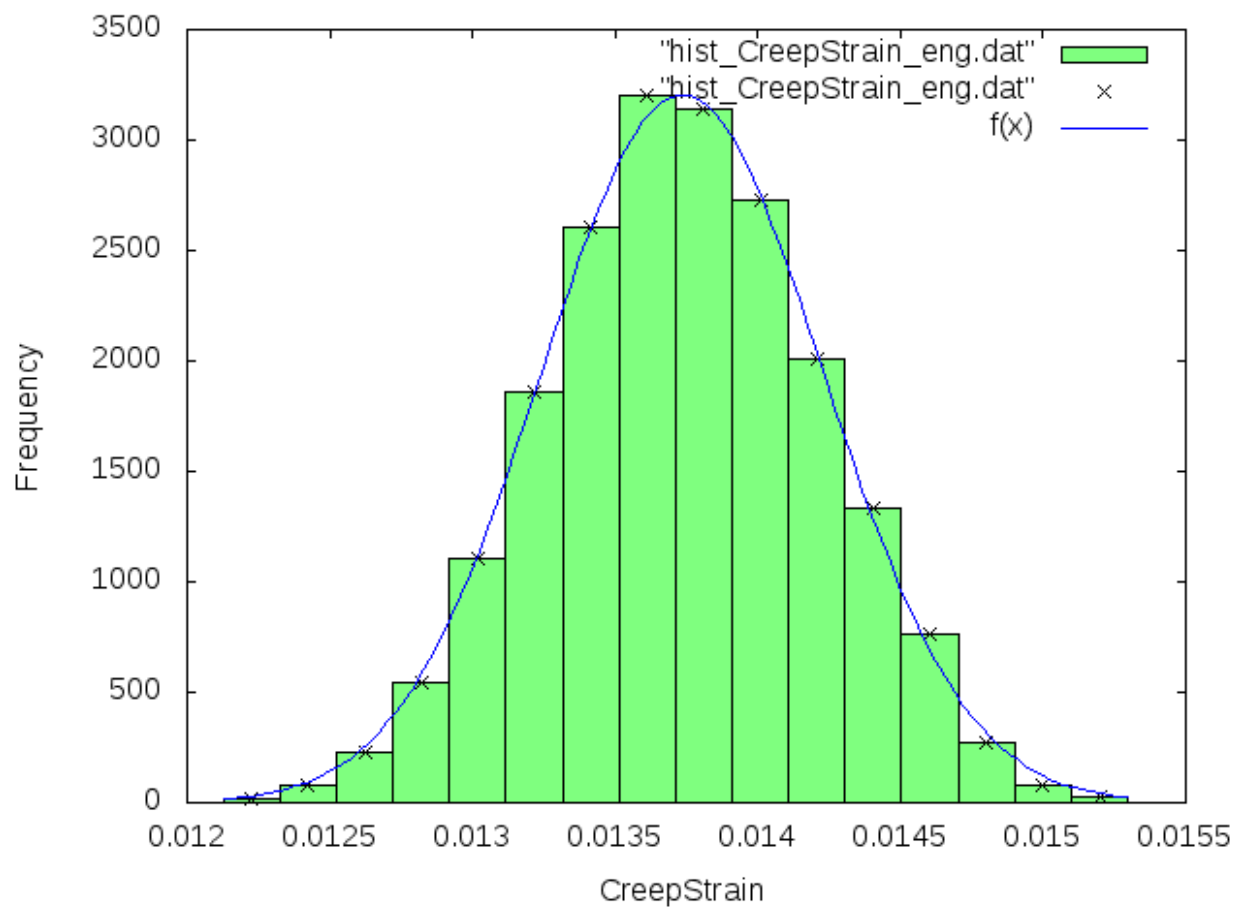

Figure 4: Distribution of cladding creep strain results (\%). 


\section{CONCLUSIONS}

The method chosen to determine the relative importance of input factors showed itself adequate, though the discrepancies between first order sensitivity indices and total effects indices suggest the number of samples in each simulation could be higher in order to offer better statistics.

For the case of TRANSURANUS code subject to abrupt changes in irradiation during the beginning of life, it was shown that fuel center temperature, fuel rod inner pressure and cladding creep strain stay well within safety limits. The input factors considered most important for the temperature are average fabrication porosity (responsible for $24 \%$ of temperature variance), porosity at the end of densification (24\%) and cladding inner radius (47\%). For the pressure, important inputs are initial fill gas pressure (92\%) and cladding inner radius $(6 \%)$. For the creep strain, cladding inner and outer radius were responsible for $13 \%$ and $30 \%$ of variance, respectively; other important factors were filling gas pressure (25\%), average porosity (13\%) and porosity at the end of densification (21\%).

Based on those results, TRANSURANUS models for the relevant outputs are considered additive, and no input interactions (second order effects) are present. 


\section{REFERENCES}

[1] LOUCKS, P. et al. Water Resources Systems Planning and Management: An Introduction to Methods, Models and Applications. Published by UNESCO, Paris, 2005.

[2] SAlTELLI, A. et al. Global Sensitivity Analysis: The Primer. John Wiley \& Sons, 2008.

[3] JRC “TRANSURANUS handbook”. European Commission Jrc Institute For Transuranium Elements. Karlsruhe, Germany. January 2012.

[4] Accident Tolerant Fuel Concepts for Light Water Reactors, IAEA-TECDOC-1797, IAEA, Vienna, 2016.

[5] GIOVEDI, C. et al. Fuel Performance of Iron-Based Alloy Cladding Using Modified Transuranus Code , XXI ENFIR, Santos, 2019

[6] GIOVEDI, C. et al. Assessment of stainless steel 348 fuel rod performance against literature available data using TRANSURANUS code. EPJ Nuclear Sci. Technol. 2 27, 2016.

[7] BOULORÉ, A.; STUZIK, C.; GAUDIER, F. Uncertainty and sensitivity analysis of the nuclear fuel thermal behavior. Commissariat à l'Énergie Atomique (CEA), France. Nuclear Engineering and Design, 2012.

[8] GAMBLE, K. A.; SWILER, L. P.; Uncertainty quantification and Sensitivity analysis applications to fuel performance modeling. United States: American Nuclear Society - ANS, 2016.

[9] IKONEN, T.; TULKKI, V.; Importance of input interactions in the uncertainty and sensitivity analysis of nuclear fuel behavior. Nuclear Engineering and Design, v.275, 2014.

[10] MYERS, J.L.; WELL, A. D.; Research Design and Statistical Analysis (2nd ed.). Lawrence Erlbaum, 2003. 